\title{
O projeto terapêutico singular como estratégia de organização do cuidado nos serviços de saúde mental
}

\section{The singular therapeutic project as a strategy of organization for care in mental health services}

\author{
Andréa Cristina S. Boccardo ${ }^{1}$, Fabiana Cristina Zane ${ }^{2}$, \\ Suréia Rodrigues ${ }^{3}$, Elisabete Ferreira Mângia ${ }^{4}$
}

BOCCARDO, A. C. S.; ZANE, F. C.; RODRIGUES, S.; MÂNGIA, E. F. O projeto terapêutico singular como estratégia de organização do cuidado nos serviços de saúde mental. Rev. Ter. Ocup. Univ. São Paulo, v. 22, n. 1, p. 85-92, jan./abr. 2011.

RESUMO: Este trabalho teve como objetivo compreender e discutir a importância da construção de projetos terapêuticos como estratégia de organização do cuidado nos serviços de saúde mental e identificar os princípios e diretrizes essenciais para a sua operacionalização. Trata-se de um estudo teórico apoiado na literatura nacional, foram selecionados 22 textos que respondiam aos objetivos gerais e específicos do projeto. A análise do material resultou na elaboração de três agrupamentos temáticos: conceitos sobre o projeto terapêutico singular; mecanismos e condições necessárias para o seu desenvolvimento. Os resultados mostraram que, de forma geral, os conceitos de projeto terapêutico singular são homogêneos e complementares entre si e os autores tem opinião semelhante sobre sua utilização enquanto estratégia de cuidado nos serviços de saúde mental. Porém, esta forma de cuidado requer uma nova maneira de organização do trabalho em equipe e do serviço, por esta razão os autores apontam desafios para os profissionais, usuários e família na busca da efetivação do desenvolvimento de projetos terapêuticos como eixo estruturador do cuidado em saúde mental.

DESCRITORES: Serviços de saúde mental; Atenção à saúde; Serviços comunitários de saúde mental; Desenvolvimento tecnológico.

1. Terapeuta Ocupacional, coordenadora do Centro de Atenção Psicossocial II de Mococa-SP.

2. Psicóloga, coordenadora do CAPS AD de Mococa-SP.

3. Psicóloga do Cratod/SP.

4. Professora do Departamento de Fisioterapia, Fonoaudiologia e Terapia Ocupacional da FMUSP.

Endereço para correspondência: Rua Cipotânea, 51. Cidade Universitária. CEP. 05360-160. São Paulo, SP. e-mail: mangeli@usp.br 


\section{INTRODUÇÃO}

$\mathrm{N}$

o Brasil, o processo de constituição da Política de Saúde Mental, teve como caracte-

rísticas centrais: a redução progressiva dos

leitos em hospitais psiquiátricos e a expansão da rede de serviços substitutivos. Além disso, tomou como princípios a inclusão e a participação e dos usuários, familiares e comunidade no processo e a manutenção das pessoas com transtorno mental em seu contexto social. Alicerçada nos princípios e diretrizes do Sistema Único de Saúde (SUS), propõe estratégias que visam a mudança do paradigma biomédico procurando romper com a assistência voltada exclusivamente para a doença, o cuidado centrado na remissão dos sintomas e como a concepção sobre a periculosidade e incapacidade presumida da pessoa com transtorno mental. Esse conjunto de desafios implica na implementação de mudanças nas práticas assistenciais e na organização dos serviços (BRASIL, 2007).

Esta nova lógica, assumida em diversos países, propõe que os serviços substitutivos se organizem na forma de uma rede articulada e acessível que objetive o resgate de cidadania e o envolvimento dos usuários, famílias e comunidade em todas as fases do processo de cuidado. Além disso, acredita que essa rede deva se organizar de acordo com as necessidades de seus usuários e articular, concomitantemente, ações que visem à qualidade de vida e autonomia das pessoas com transtorno mental e a expansão de sua rede social e de relações (OMS, 2001; BRASIL, 2007; THORNICROFT; TANSELLA, 2010).

A idéia fundamental é que somente uma organização em rede e não apenas um serviço, é capaz de fazer face à complexidade das demandas de inclusão de pessoas secularmente estigmatizadas, em um país de acentuadas desigualdades sociais... É a articulação em rede de diversos equipamentos da cidade (...) que pode garantir resolutividade, promoção da autonomia e da cidadania (BRASIL, 2005, p.25).

Para esta concepção de rede de serviços, os Centros de Atenção Psicossocial passaram a ocupar lugar central e devem assumir a função de organizar a demanda e a própria rede de cuidados em saúde mental de seu território, que podem ser compostas por residências terapêuticas, leitos em hospital geral, centros de convivência, ações de saúde mental na atenção básica, dentre outros (BRASIL, 2004).

De acordo com as normas do Ministério da Saúde, o Centro de Atenção Psicossocial (CAPS) é um serviço de saúde comunitário aberto, que oferece atendimento diário às pessoas com transtornos mentais severos e persistentes, realiza acompanhamento clínico e reinserção social por meio do acesso ao trabalho, lazer, exercício dos direitos civis e fortalecimento de laços familiares e comunitários. Desenvolve atividades dentro e fora de seu espaço físico (BRASIL, 2004).

Nos documentos que orientam o funcionamento dos CAPS são apresentadas duas definições distintas para a proposição de projeto terapêutico: a primeira diz respeito ao projeto institucional do serviço, necessário ao seu cadastramento e funcionamento, e a segunda se refere à organização do trabalho assistencial oferecido aos usuários (BRASIL, 2004).

A implantação dos Centros de Atenção Psicossocial e da rede de serviços substitutivos de saúde mental vem sendo incentivada pelo Ministério da Saúde, porém além da necessidade de ampliação de serviços é preciso definir "novas estratégias de cuidado adequadas aos princípios e diretrizes propostas pela Política de Saúde Mental" (BRASIL, 2007; MÂNGIA; BARROS, 2009).

Nesse cenário, se coloca como central a discussão sobre a formulação e o desenvolvimento de projetos terapêuticos, pois através deles que se pode materializar e concretizar o conjunto de diretrizes propostas pela nova política e assegurar aos sujeitos um contexto assistêncial (...) capaz de promover a qualidade de vida (MÂNGIA; BARROS, 2009, p. 86).

Neste artigo será abordado o tema da construção de projetos terapêuticos como estratégia central para promover o cuidado e a organização do trabalho nos serviços substitutivos de saúde mental. Atualmente, é possível identificar que a organização de projetos terapêuticos já faz parte da rotina dos serviços de saúde mental, especialmente dos CAPS, e vem também se expandindo para outros serviços de saúde e tem se fortalecido, especialmente, nas estratégias de Saúde da Família.

Assim, este estudo buscou, a partir de análise documental, compreender e discutir a construção de projetos terapêuticos na rede de serviços de saúde mental e, mais especificamente, procurou elementos que pudessem caracterizar seus mecanismos de organização e condições para sua realização.

\section{PROCEDIMENTOS METODOLÓGICOS}

Trata-se de um estudo teórico que buscou sustentação na literatura nacional. Foram consultados teses, artigos, livros, documentos oficiais. A busca foi desenvolvida a partir de consultas as bases: SciELO, DEDALUS USP, Google acadêmico e site do Ministério da Saúde. Para a busca foram utilizadas as seguintes palavras chave: projeto terapêutico, saúde mental, CAPS, trabalho em equipe e organização do trabalho. 
A busca resultou na seleção de 63 textos, destes foram escolhidos 37 para leitura detalhada e, desses, 22 textos foram considerados adequados aos objetivos do trabalho, visando as seguintes questões norteadoras: quais são as definições sobre o projeto terapêutico?; como são propostos seus mecanismos de organização prática? e que outros elementos são necessários para a realização desta estratégia?

Do processo de leitura e pareamento com as questões norteadoras resultaram três agrupamentos temáticos que orientaram a organização e apresentação do trabalho: definição de projetos terapêuticos, mecanismos de organização do serviço para o desenvolvimento de projetos terapêuticos e condições necessárias para realização de projetos terapêuticos.

\section{RESULTADOS E DISCUSSÃO}

Os dados serão apresentados em três agrupamentos temáticos na seguinte ordem: definição de projeto terapêutico; mecanismos de organização do serviço para o desenvolvimento de projetos terapêuticos e condições necessárias para a realização de projetos terapêuticos.

\section{Definição de projeto terapêutico}

A pesquisa mostrou que os autores apresentam pontos de vista similares e complementares sobre a definição de projeto terapêutico. De um modo geral, projeto terapêutico é compreendido como uma estratégia de cuidado organizada por meio de ações articuladas desenvolvidas por uma equipe multidisciplinar e definida a partir da singularidade do indivíduo, considerando suas necessidades e o contexto social em que está inserido.

Documento orientador veiculado pelo Ministério da Saúde oferece uma definição mais ampla sobre projeto terapêutico singular, visto como um conjunto de ações terapêuticas, resultantes de discussão e construção coletiva de uma equipe multidisciplinar. É um instrumento que ultrapassa o paradigma médico, na busca de resolver as necessidades das pessoas para além do critério diagnóstico (BRASIL, 2007a).

A construção de um projeto terapêutico singular deve ser compreendida como estratégia que, em sua proposição e desenvolvimento, envolve a pessoa com transtorno mental, seus familiares e a rede social, num processo contínuo, integrado e negociado de ações voltadas à satisfação de necessidades e produção de autonomia, protagonismo, inclusão social. Além disso, esse processo, visa atingir mudanças sustentáveis, ao longo do tempo, e a redução da dependência exclusiva dos sujeitos aos serviços de saúde por meio da ativação de recursos exteriores a eles (MANGIA, 2002; MÂNGIA; MURAMOTO, 2007).

Barros (2009) propõe que os projetos terapêuticos devem superar o paradigma biomédico por meio de ações conjuntas e coletivas para responder as necessidades das pessoas, geradora de mudança de vida e dá atenção especial e recomenda a utilização da terminologia Projeto Terapêutico Singular (PTS). Proposto por diversos autores, este conceito apreende com maior precisão, as intenções gerais e específicas assumidas na construção de projetos terapêuticos, especialmente a diretriz de estar centrado nos sujeitos, em suas necessidades e contexto de vida, o que define seu caráter singular.

Na mesma direção, PTS é definido por Oliveira como um arranjo operador e gestor de cuidado, cooperação e compartilhamento de saberes centrados no usuário, "é encontro de desejos, projetos, ideologias, interesse, visão de mundo e subjetividade" (OLIVEIRA, 2007).

Em posição complementar, Mororó (2010) define projeto terapêutico como o conjunto de condutas terapêuticas articuladas, resultantes de discussão coletiva de equipe interdisciplinar, que objetiva, além da melhoria de sintomas, a ampliação da rede social e o aumento de espaços de contratualidade para modificar o curso do adoecimento.

Para Merhy (1998), o projeto terapêutico deve ser um instrumento que responda as demandas objetivas e subjetivas dos usuários e tem como objetivo a produção de sua autonomia e apropriação de seu processo de cuidado.

A leitura dos textos selecionados permitiu observar que não existem divergências nas definições encontradas. As posições apresentadas pelos autores são complementares e há um ponto de convergência central na concepção de que o resgate da cidadania é objetivo final das estratégias de cuidado e que esta finalidade só pode ser alcançada na singularização das necessidades promovida pelo projeto terapêutico. Diferenças só foram encontradas nas nomenclaturas utilizadas, embora ocorra a prevalência da utilização do termo Projeto Terapêutico Singular.

Tendo em vista tais proposições este trabalho também adotará o termo Projeto Terapêutico Singular (PTS) por sua consistência e coerência.

\section{Mecanismos para organização}

De acordo com os autores estudados, a construção do PTS requer a transformação do modelo assistencial e a reorganização dos serviços, pois a forma como estes são organizados pode facilitar ou dificultar a construção das estratégias previstas para a efetivação do PTS. Neste 
agrupamento temático, serão apresentados os mecanismos da prática que possibilitam o desenvolvimento do PTS: primeiramente, os componentes do PTS e em seguida a organização da equipe e do serviço.

\section{A. Componentes do Projeto Terapêutico Singu-}

lar

Os autores evidenciam que para que um PTS alcance os objetivos propostos há aspectos práticos que devem ser incorporados em seu desenvolvimento. De modo geral, observou-se que estes aspectos estão relacionados aos campos do habitar, do trabalho e das trocas sociais e requerem articulações práticas das redes de suporte social que possam dar suporte e/ou potencializar as ações voltadas para o sujeito (BARROS, 2009).

Nesse sentido, a Política de Saúde Mental recomenda que os PTS devam ter como meta principal a inserção social por meio de ações que envolvam educação, esporte, cultura, lazer e também serem desenvolvidos de acordo com as necessidades e possibilidades de cada usuário. De forma que suas ações ultrapassem a própria estrutura física dos CAPS e busquem articular redes de suporte social voltadas para os sujeitos que contribuam para o restabelecimento de vínculos fragilizados e/ou perdidos ao longo do processo de adoecimento (BRASIL, 2004).

Em síntese, o PTS deve ser centrado no usuário, em todas as suas necessidades e contexto, flexível às mudanças de cada momento de vida e ser reavaliado periodicamente (OMS, 2001; MÂNGIA, 2002).

A fim de garantir a elaboração e operacionalização de um PTS, Mângia et al. (2006) propõe a adoção de alguns eixos norteadores: a centralidade na pessoa, a parceria entre equipe e usuário, a articulação dos recursos do território nas ações executadas, a ênfase no contexto da pessoa, a construção compartilhada e a definição de metas com duração previamente acordada.

Para a Política de Humanização do Sistema Único de Saúde, o PTS deve compreender: a elaboração do diagnóstico, a definição de metas e a responsabilização de todos os sujeitos envolvidos na sua execução e na constante reavaliação (BRASIL, 2007a).

O projeto terapêutico deve ser elaborado e executado com a participação de toda a equipe e dos próprios usuários, ser flexível e ter metas claras estabelecidas, conforme a avaliação dos resultados alcançados (... ) ter como objetivos, o aumento do conhecimento do paciente sobre os seus problemas, a sua autonomia afetiva, material e social (BRASIL, 2007a, p.89).

Oliveira (2007) critica a construção e utilização de protocolos rígidos na operacionalização dos PTS, afirma que isto pode gerar normatizações que se distanciam da singularidade proposta para seu desenvolvimento. Porém, acredita que a utilização de um roteiro pode direcionar a equipe e evitar que esta se perca no processo. Sugere que, primeiramente, se faça um diagnóstico situacional, que vá além das condições de saúde e levante as necessidades ocultas pela demanda. Depois recomenda o desenvolvimento de negociação, com usuário e família, das metas a serem alcançadas em um determinado período de tempo. Posteriormente, se procede a divisão das responsabilidades das ações de cuidado pela equipe e finalmente, se propõe a realização de avaliações periódicas.

Observou-se que a maioria dos autores sugere a utilização de algum roteiro ou guia para nortear o desenvolvimento do PTS, esses instrumentos podem direcionar as ações, da equipe e do usuário, necessárias para que sejam atingidos os objetivos. Os autores são unânimes em considerarem que o foco da construção e desenvolvimento do PTS deva ser o usuário em sua singularidade, e que esse processo deve contemplar ainda a participação da família e da rede social desses sujeitos.

\section{B. Organização da equipe}

Identificou-se que além da definição de projeto terapêutico e de um modelo que direcione sua prática, as novas estratégias de cuidado em saúde mental necessitam que as equipes desenvolvam novas formas de organização, diferentes daquelas tradicionalmente utilizadas nos serviços ambulatoriais. Assim, de acordo com o estudo bibliográfico, serão apresentados os mecanismos propostos para a organização da equipe no desenvolvimento do PTS.

Convém lembrar que o conceito e a prática do 'trabalho em equipe' tiveram sua origem na racionalização da assistência médica e na necessidade de integração de diferentes práticas no desenvolvimento da assistência a saúde, a partir da nova concepção do processo saúde doença definida pela Organização Mundial da Saúde, que passou a incorporar as dimensões sócio culturais e coletivas envolvidas no processo saúde-doença e não mais apenas a dimensão biológica e individual. Nesse contexto o trabalho em equipe deve promover a integração das diversas disciplinas envolvidas por meio da comunicação e do compartilhamento de ações e saberes (PEDUZZI, 2001).

O trabalho em equipe deve ser baseado nas necessidades dos usuários, na integralidade das ações e na interdisciplinaridade e, para tanto são propostas mudanças na forma de organização e relações de poder entre os membros da equipe e desta com os usuários (PEDUZZI, 2007). 
O desenvolvimento do princípio da integralidade nas práticas cotidianas dos serviços de saúde se traduz na implicação da equipe em compreender e formular estratégias de modo a atender as necessidades de saúde das pessoas e da comunidade (BARROS, 2009, p.25).

Os autores estudados constroem suas formulações, sobre a organização do trabalho em equipe, necessária ao desenvolvimento e gerenciamento de PTS, partir de um modelo organizacional baseado nos conceitos de profissional de "referência" ou "equipe de referencia. Definido por Campos (1999) como "meio de valorização concreta e operacional das diretrizes de vínculo terapêutico (p.394), constitui um novo arranjo no processo de trabalho que possibilita a divisão de responsabilidades e novos padrões de relação entre equipe e usuários, horizontalizando as práticas e saberes, rompendo com a hegemonia biomédica e tendo como função a condução dos projetos terapêuticos singulares e a vinculação entre o usuário e o serviço (CAMPOS, 1999; FILIZOLA, 2008).

Nessa direção, procura-se definir o(s) técnico(s) de referência para o usuário e familiares, respeitando-se as singulares e possíveis relações vinculares estabelecidas no processo terapêutico. O dispositivo "técnico de referência (TR)" foi instituído a partir da idéia de que um ou mais profissionais do serviço devem se aproximar de maneira diferenciada dos usuários para acompanhá-los em seus projetos terapêuticos singulares. A "referência", geralmente, é composta por um profissional de nível superior em parceria com um profissional de nível médio que se responsabilizam pelo acompanhamento do PTS, com mais proximidade e diálogo com o usuário. Isso envolve, também, os contatos com familiares, a comunicação com de equipe da atenção básica e a reavaliação periódica do PTS (MÂNGIA et al., 2009).

Para Barros, a adoção do conceito e da prática do profissional/equipe de "referência", implica, além de mudanças na organização da equipe, o desenvolvimento de um novo olhar sobre o processo de cuidado, que deve envolver todos os recursos disponíveis e ampliar a disponibilidade para estar e fazer junto (BARROS, 2009).

O processo de reorganização do trabalho em equipe e a construção de uma nova cultura assistencial deve também enfrentar o desafio de superar as normas e estruturas organizacionais tradicionais e introduzir uma nova forma divisão das responsabilidades, pautada na continuidade das ações e não mais na produção de respostas isoladas, imediatistas e descontextualizadas (MORORÓ, 2007; BARROS, 2009). Para tanto, é importante que as equipes adquiram clareza sobre seus novos papéis e propiciem aos usuários a ampliação dos espaços de participação. Tal arranjo deve buscar cons- truir redes de comunicação que ancorem e contribuam para o sucesso dos projetos terapêuticos (BARROS, 2009).

Assim, nas novas práticas, o trabalho em equipe deve superar a fragmentação do conhecimento e das especificidades profissionais a fim de construir uma prática de cuidado articulada e integrada, pois só desta forma é que se consegue responder as reais necessidades das pessoas. A organização da equipe a partir do conceito de "referência", possibilita o atendimento diferenciado e singularizado das demandas dos usuários, a melhoria da acessibilidade aos serviços, recursos e profissionais e a responsabilização de todos no processo do cuidado, na medida em que compartilha e descentraliza $\mathrm{o}$ atendimento clínico.

\section{Organização do serviço}

Os autores estudados apresentam opiniões semelhantes em relação aos mecanismos necessários para a organização do PTS nos serviços. Acolhimento, vínculo e responsabilização são as estratégias centrais para essa construção, pois são consideradas competências gerais de todos os profissionais que atuam nos serviços (MORORÓ, 2007; OLIVEIRA, 2007; MÂNGIA, 2009).

$\mathrm{O}$ acolhimento consiste na oferta de escuta humanizada direcionada para além da demanda apresentada pelo usuário e na disponibilidade para aumentar a proximidade ao longo do processo de acompanhamento. Os serviços devem desenvolver estratégias de acolhimento em todas as fases do processo de cuidado e não apenas nos momentos de crise ou de ingresso dos usuários nos serviços, pois o acolhimento proporciona espaços de trocas, escuta, manifestação de desejos e necessidades. Sem acolhimento há dificuldade de estabelecer vínculo e sem a criação de vínculo o processo de cuidado do usuário é prejudicado (FRANCO, 1999).

$\mathrm{O}$ vínculo, compreendido como: relação contínua no tempo, pessoal, intransferível e calorosa (MORORÓ, 2007, p.80) se coloca como o elemento do processo de cuidado que, a partir do acolhimento reorganiza o processo de trabalho e o funcionamento do serviço. O desenvolvimento de vínculos fortes e duradouros se coloca como objetivo para toda a equipe que acolhe, escuta e oferece respostas, garantindo a acessibilidade e um atendimento baseado nos princípios da humanização e da cidadania (FRANCO et al., 1999).

Na construção do projeto terapêutico se faz um exercício de síntese de competências, em particular da articulação entre a competência genérica de qualquer profissional de saúde como um cuidador e seu agir específico profissional, com sua competência específica diante de certa teoria sobre os modos de adoecer e construir intervenções restauradoras (MERHY, 1999, p.2). 
Nunes et al. (2008) identificaram a coexistência de três concepções de cuidado em um Centro de Atenção Psicossocial: o modelo biomédico humanizado centrado na psicopatologia caracterizado pelo cuidado assistencialista e por vezes tutelar, que objetiva a reinserção social da pessoa com transtorno mental por meio da adequação de comportamento; o modelo psicossocial com ênfase no território, onde se valoriza os aspectos sociais no processo de adoecimento, de forma que as estratégias de cuidado são vinculadas aos aspectos do trabalho, da família, e da comunidade $e$, finalmente o modelo psicossocial com ênfase na instituição, cuja concepção de cuidado que visa a integralidade e contempla os aspecto psicossociais, embora com ações restritas ao contexto institucional.

Esta contribuição revela que, embora as intervenções utilizem de estratégias comuns: escuta, diálogo, troca, produção de subjetividade, acolhimento, dentre outras, o mesmo sujeito pode ser compreendido e abordado de formas diversas, no contexto de um mesmo serviço. Tal constatação demonstra a necessidade de diálogo e de compartilhamento de saberes entre os membros da equipe, buscando a construção de um padrão homogêneo e coerente do cuidado, pois quando profissionais, de uma mesma equipe, apresentam concepções distintas, pode ocorrer conflito entre orientações e impedimentos para a prática interdisciplinar, com prejuízo para as ações terapêuticas e para os usuários (FILIZOLA, 2008).

Destaca-se que os serviços que compõem a rede de saúde mental são públicos e como tal devem garantir o acesso, a integralidade e resolutividade em todos os níveis de assistência, desta forma, o trabalho em equipe deve favorecer a organização do serviço para que este responda aos princípios e diretrizes do Sistema Único de Saúde.

Finalmente, observou-se que todos os autores julgam que para a construção de projetos terapêuticos a organização do serviço deve contemplar estratégias para o desenvolvimento das práticas de acolhimento, responsabilização e fortalecimento de vínculos. Destacam também que a estratégia de acolhimento, deve ser vista como espaço de escuta, trocas e manifestação dos diferentes aspectos que influenciam na percepção do processo de adoecimento e do entendimento do contexto de vida.

\section{CONDIÇÕES PARA REALIZAÇÃO DO PROJETO TERAPÊUTICO}

Para que as práticas empreendidas no desenvolvimento de um projeto terapêutico singular se integrem nas dimensões de organização do serviço e do trabalho da equipe são necessárias condições, que serão aqui apresentadas, sem as quais não há transformação das tecnologias de cuidado.

Barros (2009) sugere que as reuniões de equipe sejam a base de organização dos serviços e que devam contemplar espaços para discussões de casos e decisões conjuntas. Os profissionais envolvidos devem desenvolver maior clareza sobre os papéis que desempenham na equipe e junto aos usuários sob seus cuidados. Os prontuários compartilhados e com registros atualizados e compreensíveis a todos são também destacados como facilitadores do desenvolvimento de um PTS. Da mesma forma, os espaços de discussão entre usuários, família, equipe e a interlocução da equipe com outros serviços envolvidos no cuidado e as reavaliações sistemáticas são fundamentais no desenvolvimento de cada PTS.

As reuniões de equipe devem ser organizadas como espaços de reflexão, discussão, compartilhamento e divisão de tarefas e responsabilidades, promovendo horizontalização do conhecimento, para que todos na equipe sintam-se membros e responsáveis (LEÃO; BARROS, 2008; FILIZOLA et al., 2008; NUNES et al., 2008).

O trabalho em equipe propicia a integração das ações dos profissionais e é essencial para o novo modelo de atenção proposto, porém os autores evidenciam a dificuldade deste tipo de organização, uma vez que algumas equipes ainda estão centradas no modelo biomédico e apresentam dificuldade de comunicar-se e compartilhar os saberes (ADUHAB et al.; BRASIL, 2007).

Barros identifica como obstáculos para o desenvolvimento de PTS: a dificuldade da equipe em identificar a base teórica de sua prática; sobrecarga de responsabilidade assistencial ocasionada pela alta demanda; falta de qualificação da equipe e a dinâmica proposta para as reuniões. A forma de organização das equipes de referência, pode também dificultar a troca de informações e a coordenação necessária para a organização do trabalho e definição de metas e prioridades das ações em um PTS (BARROS, 2009).

Da mesma forma, são considerados obstáculos: a falta de espaços para discutir o PTS com o usuário e família; a dificuldade da equipe em se dispor a compreender e atender as necessidades do usuário; a fragmentação do desenvolvimento do PTS nas etapas de prevenção, tratamento e reabilitação ao invés de uma concepção contínua e integrada entre esses aspectos; a falta ou insuficiência de registros em prontuários; a formação profissional inadequada para as necessidades da nova política assistencial e a rotatividade da equipe (Barros, 2009). Se acrescenta a esses aspectos: a falta ou insuficiência de comunicação da equipe; o uso de modelos fechados e a compreensão do PTS como um mero dispositivo administrativo (MORORÓ, 2010; NUNES et al., 2008).

Assim, em relação às condições necessárias para 
o desenvolvimento de um PTS, o estudo mostrou pontos de vista comuns e complementares: as reuniões de equipe como espaço central de reflexão das práticas e de produção de conhecimento, no contexto da adoção dos princípios da integralidade, interdisciplinaridade e horizontalização do saber são condições indissociáveis ao desenvolvimento das novas práticas em saúde mental.

\section{CONSIDERAÇÕES FINAIS}

A implantação dos serviços de saúde mental, em especial os Centros de Atenção Psicossocial (CAPS), colocam no processo de cuidado o contexto e a história de vida dos usuários, rompendo com o modelo biomédico hegemônico que distancia a singularidade dos sujeitos e sua subjetividade do processo de tratamento e reabilitação.

As transformações do modelo assistencial atribuem aos serviços de saúde mental, à equipe, aos usuários e seus familiares novas funções compartilhando responsabilidades a todos os atores envolvidos no processo de cuidado. Neste contexto, o desenvolvimento de projetos terapêuticos singulares se constitui como estratégia central de produção do cuidado e visa promover acolhimento, vínculo e responsabilização pelos usuários e garantir atenção continuada e integral. Por meio da escuta e do "estar junto" promovidos pelo acolhimento a equipe pode desenvolver habilidades capazes de tornar o cuidado mais adequado às necessidades das pessoas com transtorno mental.

As mudanças na organização dos serviços e equipes, promovidas pela organização de projetos terapêuticos singulares, transformam as práticas de cuidado e também os papéis da equipe ao modificarem o foco da doença e da remissão de sintomas para a centralidade nas necessidades das pessoas em seu contexto social.

Um dos grandes desafios dos CAPS e demais serviços de saúde mental é promover o cuidado integral por meio de ações que atendam as reais necessidades dos usuários, além da reorganização do serviço e da equipe para atender as novas responsabilizações fomentadas pela Política de Saúde Mental. Nesse sentido vê-se como fundamental a necessidade da organização do trabalho em equipe multiprofissional e interdisciplinar que possa dar suporte ao novo modelo, no desenvolvimento de suas práticas e na produção de conhecimento.

Por fim, se concluí que a adoção de modelos para a produção projetos terapêuticos nos serviços de saúde mental é um processo em construção, especialmente nos CAPS e não tem sido uma tarefa fácil para todos os atores envolvidos na medida que todo processo em construção gera conflitos, ambigüidades e dúvidas.

BOCCARDO, A. C. S.; ZANE, F. C.; RODRIGUES, S.; MÂNGIA, E. F. The singular therapeutic project as a strategy of organization for care in mental health services. Rev. Ter. Ocup. Univ. São Paulo, v. 22, n. 1, p. 85-92, jan./abr. 2011.

\begin{abstract}
S: This work piece had as objective to understand and discuss the importance of the construction of therapeutic projects as an organization axis in mental health care and identify the principles and essential guideline to its accomplishment. It is a matter of a theoretical study based on national literature, there had been selected 22 extracts that answered general and specific objectives of this work piece. The analysis of this material resulted in the development of three theme groupings: concepts about the singular therapeutic project; mechanisms and necessary conditions to its development. The results showed that, in general, the concepts of the singular therapeutic project are homogeneous and complementary among them and the authors have a similar opinion about its use as strategy for care in mental health services. However, this way of care requires a new way of working organization in teams and service, as a matter of fact the authors point out the challenges for the professionals, users and family in search for the effectiveness in the development of therapeutic projects as a structural axis of care in mental health.
\end{abstract}

KEY WORDS: Mental health services; Health care (Public health); Community mental health services; Technologic development.

\title{
REFERÊNCIAS
}

ADUHAB, D.; SANTOS, A. B.; MESSENBERG, C. B.; FONSECA, R. M. G. S.; ARANHA E SILVA, A. L. O trabalho multiprofissional no Caps III: um desafio. Revista Gaúcha de Enfermagem, Porto Alegre; v. 26, n. 3, p. 369-380, 2005.
BARROS, J. O. A construção de projetos terapêuticos no campo da saúde mental: apontamentos acerca das novas tecnologias de cuidado. São Paulo, 2009. [Dissertação]. Faculdade de Fisioterapia, Fonoaudiologia e Terapia Ocupacional da FMUSP, 
Universidade de São Paulo.

BRASIL. Ministério da Saúde. Equipe ampliada, equipe de referência e projeto terapêutico singular. $2 \mathrm{a}$. ed. Série textos básicos de saúde. Brasília, DF, 2007a.

BRASIL. Ministério da Saúde. Secretária de Atenção à Saúde/ DAPE. Saúde mental no SUS: acesso ao tratamento e mudança no modelo de atenção. Relatório de gestão 2003-2006. Brasília, 2007.

BRASIL. Ministério da Saúde. Secretaria de Atenção à Saúde. Departamento de Ações Programáticas Estratégicas. Coordenação Geral de SM. Saúde no SUS: os centros de atenção psicossocial. Brasília, 2004.

BRASIL. Ministério da Saúde. Secretaria de Atenção à Saúde/ DAPE. Coordenação Geral de Saúde Mental. Reforma Psiquiátrica e política de saúde mental no Brasil. Documento apresentado à Conferência Regional de Reforma dos Serviços de Saúde mental 15 anos depois de Caracas. Brasília, 2005.

CAMPOS, G. W. S. Equipes de referência e apoio especializado matricial: um ensaio sobre a reorganização do trabalho em saúde. Revista Ciencias e Saúde Coletiva, v. 4, n. 2, p. 393-403, 1999.

FILIZOLA, C. L. A.; MILIONI, D. B.; PAVARINI, S. C. I. A vivência dos trabalhadores de um CAPS diante da nova organização do trabalho em equipe. Revista Eletrônica de Enfermagem, v. 26, n.3, p. 491-503, 2008. disponível em: < http://www.fen.ufg.br/ revista/v10/n2/pdf/v10n2a20.pdf $>$.

FRANCO, T. B., BUENO, W. S.; MERHY, C. C. O acolhimento e os processos de trabalho em saúde: o caso de Betim, Minas Gerais, Brasil. Cadernos de Saúde Pública, v. 15, n. 2, 1999. Disponível em: < http://www.scielosp.org/pdf/csp/v15n2/0319.pdf>.

LEÃO, A.; BARROS, S. As representações sociais dos profissionais de saúde mental acerca do modelo de atenção as possibilidade de inclusão social. Revista Sociedade, São Paulo, v. 17, n. 1, p. 95-106, 2008.

MÂNGIA, E. F.; BARROS, J. O. Projetos terapêuticos e serviços de saúde mental: caminhos para a construção de novas tecnologias de cuidado. Revista de Terapia Ocupacional da Universidade de São Paulo, v. 20, n. 2. p. 85-91, 2009.

MÂNGIA, E. F.; CASTILHO, J. P. L. V.; DUARTE, V. R. E. D. A construção de projetos terapêuticos: visão de profissionais em dois Centros de Atenção Psicossocial. Revista de Terapia Ocupacional da Universidade de São Paulo, v. 17, n. 2, p. 87-98, 2006.

MÂNGIA, E. F.; MURAMOTO, M. T. Redes sociais e construção de projetos terapêuticos: um estudo em serviço substitutivo em saúde mental. Revista de Terapia Ocupacional da Universidade de São Paulo, v. 18, p. 54-62, 2007.

MÂNGIA, E. F. Contribuições da abordagem canadense "Prática de Terapia Ocupacional Centrada no Cliente" e dos autores da desinstitucionalização italiana para a Terapia Ocupacional em saúde mental. Revista de Terapia Ocupacional da Universidade de São Paulo, v. 13, n. 3, p.127-34, 2002.

MERHY E. E. A perda da dimensão cuidadora na produção da saúde: uma discussão do modelo assistencial e da intervenção no seu modo de trabalhar a assistência. In: REIS, A. T.; Santos, A. F.; CAMPOS, C. R.; MALTA, D. C.; MERHY, E. E. (Orgs.). Sistema Único de Saúde em Belo Horizonte: reescrevendo o público. São Paulo: Xamã, 1998. p. 103-120, 1998.

MERHY, E. E. Apostando em Projetos Terapêuticos Cuidadores: desafios para a mudança da escola médico ou utilizando-se da produção dos projetos terapêuticos em saúde como dispositivo de transformação das práticas de ensino-aprendizagem que definem os perfis profissionais dos médicos. Campinas, 1999. Disponível em: http//www.uff.br/saudecoletiva/professores/merhy/artigos-07.pdf

MORORÓ, M. E. M. L. Cartografias, desafios e potencialidades na construção de projeto terapêutico em Centro de Atenção Psicossocial. [Dissertação]. São Paulo, 2010. Escola de Enfermagem da Universidade de São Paulo.

NUNES, M.; TORRENTÉ, M.; OTONI, V.; MORAES NETO, V.; SANTANA, M. A dinâmica do cuidado em saúde mental: signos, significados e práticas de profissionais em um Centro de Assistência Psicossocial em Salvador, Bahia, Brasil. Cadernos de Saúde Pública, Rio de Janeiro, v. 24, n. 1, p. 188-196, 2008.

OLIVEIRA, G. N. O projeto Terapêutico como contribuição para a mudança das práticas de saúde. [Dissertação]. Campinas, 2007. Faculdade de Ciências Médicas, Universidade Estadual de Campinas.

ORGANIZAÇÃO MUNDIAL DE SAÚDE (OMS). Organização Pan-Americana de Saúde (OPAS). Relatório sobre a saúde no mundo 2001. Saúde mental, nova concepção nova esperança. Genebra: Gráfica Brasil, 2001.

PEDUZZI, M. Trabalho em equipe de saúde no horizonte normativo da integralidade, do cuidado e da democratização das relações de trabalho. In: PINHEIRO, R.; BARROS DE BARROS, M. E.; MATTOS, R. A. Trabalho em equipe sob o eixo da integralidade: valores, saberes e práticas. Rio de Janeiro: CEPESC/ABRASCO, 2007. p. 161-178.

PEDUZZI, M. Equipe multiprofissional de saúde: conceito e tipologia. Revista Saúde Pública, v. 35, n. 1, p.103-109, 2001.

THORNICROFT, G.; TANSELLA, M. Boas práticas em saúde mental comunitária. Barueri (SP): Manole, 2010.

Recebido para publicação: 11/03/2011

Aceito para publicação: 01/04/2011 